\title{
Large Solar Flare
}

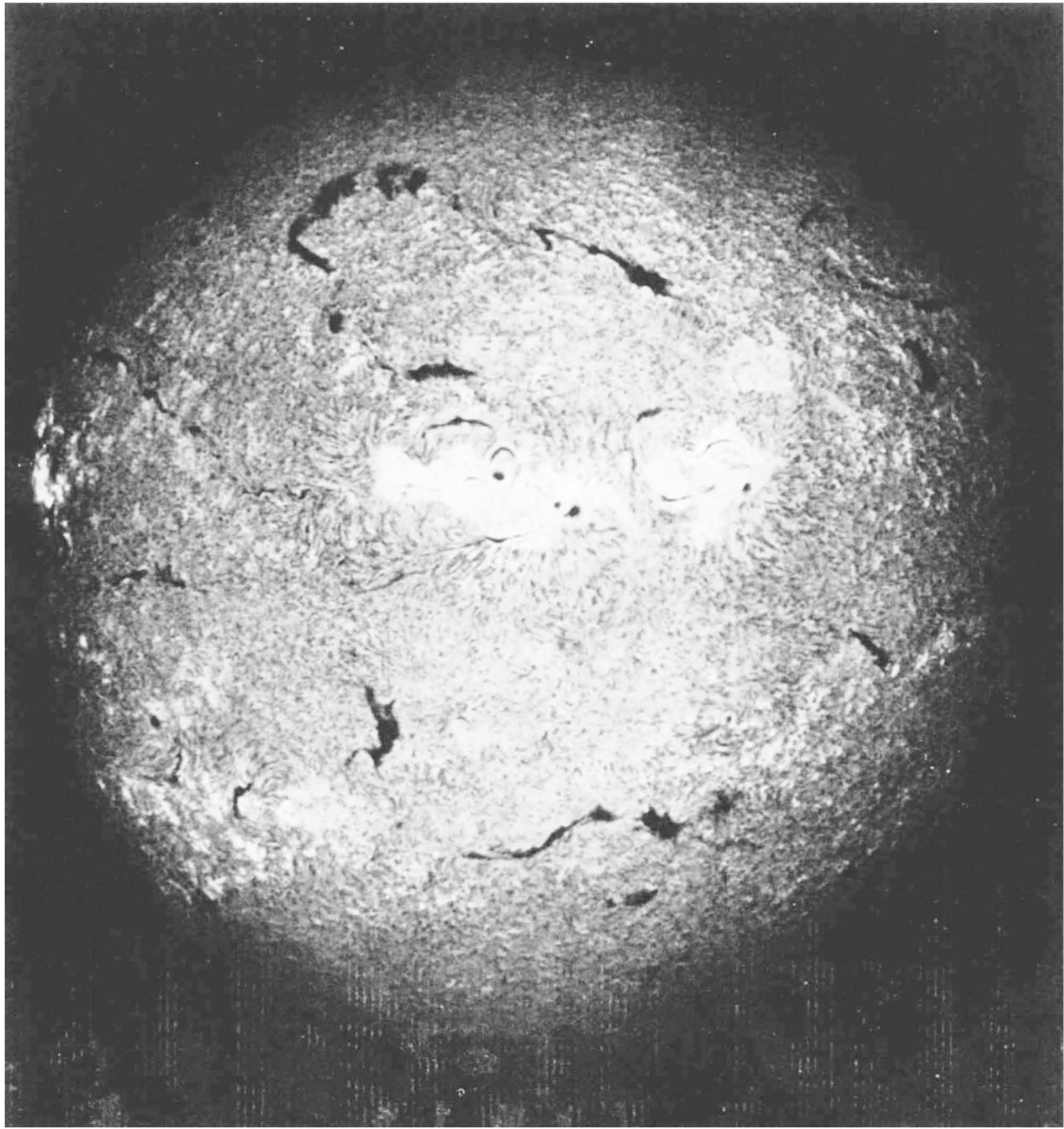

Astronomers at Pennsylvania State University, who took this photograph of the Sun on July 12 at 1421 UT, say that it shows one of the largest solar flares ever recorded. The photograph was taken in the light of the $\mathrm{H}_{\alpha}$ line $(6562.8 \AA)$, with a half width of $0.5 \AA$, using a new telescope recently installed at Pennsylvania State University and fitted with a Zeiss monochromator. It shows solar prominences as narrow black filaments, strongly absorbing $\mathrm{H} \alpha$, and the white swirls known as plages, which are often associated with sunspots. The flare was on the right of the two plages near the centre of the photograph. Events such as this, which disrupted shortwave radio communications on July 12, should become more common during the next few months, as the Sun approaches the maximum of its eleven year cycle of activity.

The programme at Pennsylvania State University is to include measurements of the movement of solar eruptions. This is done by slightly shifting the passband of the monochromator from the $\mathrm{H} \alpha$ line. If the eruption is moving away from the Sun, toward the Earth, a more intense photograph of the eruption will be obtained with the pass-band shifted to the blue side of the $\mathrm{H} \alpha$ line. On the other hand, if it is falling back on to the Sun's surface, a photograph on the red side of the $\mathrm{H} a$ line will be the more intense. 